\section{Limiting factors for modellers}

\author{
J. P. Grime
}

Plant Strategies and the Dynamics and Structure of Plant Communities. By David Tilman. Princeton University Press:1988. Pp.360. Hbk \$45; pbk \$15.95.

ThOSE attempting to model the full richness of vegetation dynamics must grapple with many facets of ecological research. They must recognize that each relevant field has its own concepts, and linkages must be established between some traditionally disparate fragments of the 'ecological jigsaw'. All embarking on such a venture must brace themselves against the natural reactions of specialists who see their work simplified in the cause of generality

A further hazard is that the author of such an ambitious model is likely to introduce an unconscious bias promoting ideas derived from his own research experience and education. David Tilman has not escaped from this problem, and the strengths and weaknesses of his latest book are closely related to his origins as a modeller of the Princeton school and as a student of phytoplankton. In ALLOCATE, the simulation model which lies at the heart of his analysis, we find a bold attempt to identify the selection forces and constraints which dictate ecological specialization in plants. The result is an admirable but seriously flawed attempt to replace word models with mathematics and to test predictions against experimental data.

Tilman believes that competition is almost universally important as a determinant of ecological success, and he explains spatial variation in vegetation in terms of the differing abilities of species to tolerate particular resource shortages. The notion of relative tolerance as the mechanism of competition is contentious and at odds with the views of both Charles Darwin (as expressed in The Origin of the Species) and A. Milne (in Mechanisms in Biological Competition, edited by F.L. Milthorpe; Cambridge University Press, 1961). The origins of Tilman's model are readily apparent in his studies of the behaviour of mixed phytoplankton populations, where the struggle for existence frequently involves relative tolerance of resource shortages. Setting aside the vexed question of whether relative tolerance can be equated with competition, there are difficulties in extrapolating the model to terrestrial vegetation, in that equilibration time must be extended from days (phytoplankton) to more than 100 years (forest succession).

Tilman recognizes this limitation and introduces the notion of "transient dynamics" to describe the floristic changes which may occur over many years before equilibrium is reached; this is a major concession and encourages the hope that ALLOCATE will evolve further towards ecological reality. In its present form, however, the model is severely restricted in general relevance, not only by its dependence upon equilibrium conditions of resource limitation, but by the prime importance which is given to the partitioning of resources between root and shoot. This reflects the author's assumptions about the availability of light and soil resources in various habitats. Tilman notes the conspicuous shaded stratum of dense productive vegetation and interprets this as evidence of light-limitation. But he fails to acknowledge the large body of literature which documents less obvious but equally important factors, such as the expanding zones of water and nutrient exhaustion which accompany root activity during each growing season. This patchiness and the attendant foraging responses of the roots of the fast-growing plants of

\section{The real thing}

\section{Murray Stewart}

Artifacts in Biological Electron Microscopy. Edited by Richard F. E. Crang and Karen L. Klomparens. Plenum:1988. Pp.233. In the United States \$45, elsewhere \$54.

READING a book on artefacts in electron microscopy is rather like reading the Karma Sutra: one begins in a frenzy of anticipation and ends with a sense of amazement as to what people can do. In both instances one is presented with an intriguing catalogue of techniques that is useful for both instruction and inspiration, but that also contains quite a bit of information that may not be all that relevant in everyday life. And there is just so much that one is interested in that is not discussed at all.

Many chapters are excellent. Considerable attention is devoted to the difficulties encountered in sectioned embedded material; given the large number of cell biologists who now use these methods without being aware of the pitfalls that were common knowledge in the 1960 s and 1970 s, the inclusion of this topic was particularly appropriate. The chapters on scanning microscopy pinpoint many of the problems that crop up and describe ways of overcoming them. There is, too, a chapter on photography. Although the author's purpose is to provide advice as to how to record and print micrographs, this is nonetheless a fascinating article.

Other contributions are less worthwhile. The treatment of instrumentinduced artefacts in transmission microscopy is rudimentary, and there is only the fertile soils, studied by M.C. Drew and others, strongly suggest that competitive ability at its most potent is an interactive property in which high rates of capture of all resources are sustained by simultaneous foraging responses which enable escape from depletion zones both above and below ground.

Tilman begins with the stated aim of exploring "the mechanisms of competition for soil resources and light". It is therefore disappointing to find that his model's dependence upon equilibration has necessitated a definition of competition which merges the process with a multiplicity of other factors determining the relative fitness of neighbouring populations. This is clearly unsatisfactory. As Milne observed, "competition ought to have only one meaning - clear, precise and unambiguous".

J.P. Grime is a Professor in the Natural Environment Research Council Unit of Comparative Plant Ecology, University of Sheffield, Sheffield S10 2TN, UK. most cursory account (often misleading if not actually incorrect) of image-formation mechanisms and focus-related artefacts. There is no real consideration of the compromise between resolution and contrast that has to be made with most biological material, or of the key importance of radiation damage in recording finer features or in making 3-D reconstructions from tilt series. The treatment of electron probe X-ray microanalysis is also too superficial to be really useful.

Quite astonishingly there is nothing on artefacts associated with negative staining or shadowing, both of which are extremely widely used, while most modern methods are either ignored or mentioned in asides. Such an important new technique as cryoelectron microscopy is discussed in terms of frozen sections, the extensive use of this method for observing material frozen in films of vitreous ice (an extremely fertile source of artefacts) being dealt with only in passing. Moreover, image processing, which is so powerful in obtaining reliable information especially from regular objects, is not included at all. That is a great pity - a discussion of artefacts in image processing of electron micrographs really would be something.

So, perhaps rather like the Karma Sutra, this book leaves one's interest aroused rather than satisfied, and wishing that, in places, it had been more explicit and that greater emphasis had been put on more up-to-date techniques. It nonetheless provides instructive reading for cell biologists, particularly those who only occasionally use electron microscopy.

Murray Stewart is in the Division of Structural Studies, Medical Research Council Laboratory of Molecular Biology, Hills Road, Cambridge CB2 $2 Q H, U K$ 